04

\title{
Нелинейная теория обратных и боковых ионно-звуковых волн в плазме с однонаправлено движущимися ионами
}

\author{
(C) A.Е. Дубинов, И.Н. Китаев
}

Российский фредеральный ядерный центр - Всероссийский научно-исследовательский институт экспериментальной фозики,

607188 Саров, Россия

Национальный исследовательский ядерный университет - Московский инженерно-физический институт, 115409 Москва, Россия

Саровский фризико-технический институт НИЯУ МИФИ,

607186 Саров, Россия

e-mail: rmtmkitaeva@yandex.ru

Поступило в Редакцию 31 марта 2019г.

В окончательной редакции 31 марта 2019г.

Принято к публикации 3 июля 2019 г.

\begin{abstract}
Рассмотрены наклонные ионно-звуковые волны в плазме, в которой ионы движутся однонаправлено. В рамках линейной теории выведено двумерное дисперсионное соотношение и показано, что в системе возможны обратные и боковые волны. Развитая в работе нелинейная теория обратных и боковых волн базируется на выводе и анализе псевдопотенциала Сагдеева. Вычислены нелинейные профили обратной и боковой ионно-звуковых волн. Найдено, что профили обратной и боковой волн неотличимы от профиля обычной прямой ионно-звуковой волны.
\end{abstract}

Ключевые слова: плазма, ионно-звуковая волна, обратная волна, боковая волна, псевдопотенциал Сагдеева.

DOI: 10.21883/JTF.2020.01.48660.134-18

\section{Введение}

Обратными волнами называются волны, в которых фазовая и групповая скорости параллельны, но направлены в разные стороны. Прямыми волнами называются волны с сонаправленными фазовой и групповой скоростями. И, наконец, боковыми волнами называются волны, в которых фазовая и групповая скорости не параллельны друг другу. При этом угол между фазовой и групповой скоростью в боковой волне называется углом сноса.

В работе [1] в рамках одномерной теории было показано, что в незамагниченной плазме, в которой ионы движутся однонаправлено, ионно-звуковые волны могут принимать форму обратных волн. Аналогично в [2] показано, что пылеакустические волны в запыленной плазме также могут быть обратными, если пылевой поток движется сквозь плазму.

В работе [3] в рамках двумерной теории показано, что ионно-звуковые волны, распространяющиеся в плазме под углом ионному потоку, могут быть не только прямыми или обратными, но и боковыми.

В [1-3] рассмотрения проводились в рамках линейного приближения, когда выводились и анализировались дисперсионные соотношения для волн. Нелинейная теория обратных и боковых волн не рассматривалась.

Отметим, что линейные и нелинейные ионно-звуковые волны в плазме с ионным потоком рассматривались ранее в работах [4-8], однако вопросы существования обратных и боковых волн там не затрагивались.
В связи с этим целью настоящей работы является рассмотрение боковых нелинейных ионно-звуковых волн в плазме с однонаправлено движущимися ионами.

\section{Линейная теория}

Рассмотрим идеальную бесстолкновительную незамагниченную электрон-ионную плазму, в которой электронную компоненту вследствие малой массы частиц будем считать безынерционной и подчиняющейся распределению Больцмана

$$
n_{e}(\varphi)=n_{0 e} \exp \left(\frac{e \varphi}{k_{\mathrm{B}} T_{e}}\right)
$$

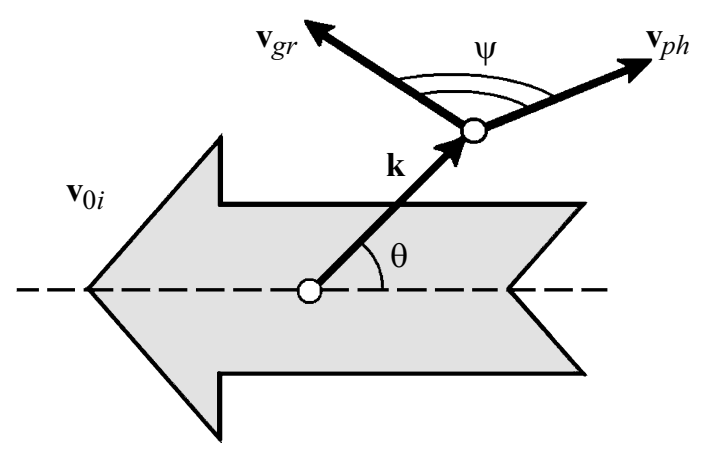

Рис. 1. Геометрия задачи. 
а ионную компоненту - подчиняющейся уравнению состояния идеального газа

$$
p_{i}=n_{i} k_{\mathrm{B}} T_{i},
$$

причем для простоты остановимся на рассмотрении изотермического случая $V_{T_{i}} \neq 0, T_{i}=$ const. Кроме того, допустим, что ионы однократно заряжены, и что они однонаправлено движутся со скоростью $\mathbf{v}_{0 i}$. В (1) и (2) обозначено: $n_{e, i}$ - концентрации электронов и ионов в волне, $T_{e, i}-$ их температуры, $n_{0 e}-$ невозмущенная концентрация электронов, $\varphi$ - электростатический потенциал, $k_{\mathrm{B}}-$ постоянная Больцмана, $p_{i}$ - давление ионного газа. Геометрия задачи показана на рис. 1.

Будем исходить из следующих уравнений ионной динамики:

$$
\begin{gathered}
\frac{\partial n_{i}}{\partial t}+\nabla\left(n_{i} \mathbf{v}_{i}\right)=0 \\
\frac{\partial \mathbf{v}_{i}}{\partial t}+\left(\mathbf{v}_{i} \nabla\right) \mathbf{v}_{i}=-\frac{e}{m_{i}} \nabla \varphi-\frac{1}{m_{i} n_{i}} \nabla p_{i} \\
\Delta \varphi=4 \pi e\left(n_{e}-n_{i}\right)
\end{gathered}
$$

где $e$ - элементарный заряд (считается, что ионы однозарядные), $m_{i}$ - масса иона, $\mathbf{v}_{i}-$ скорость ионов.

В двумерной геометрии уравнения (3)-(5) можно записать в скалярной форме, выбирая систему координат так, чтобы ионный поток был направлен вдоль оси $0 x$ :

$$
\begin{gathered}
\frac{\partial n_{i}}{\partial t}+\frac{\partial\left(n_{i} v_{x i}\right)}{\partial x}+\frac{\partial\left(n_{i} v_{y i}\right)}{\partial y}=0 \\
\frac{\partial v_{i x}}{\partial t}+v_{i x} \frac{\partial v_{i x}}{\partial x}+v_{i y} \frac{\partial v_{i x}}{\partial y}=-\frac{e}{m_{i}} \frac{\varphi}{\partial x}-\frac{k_{\mathrm{B}} T_{i}}{m_{i}} \frac{1}{n_{i}} \frac{\partial n_{i}}{\partial x} \\
\frac{\partial v_{i y}}{\partial t}+v_{i x} \frac{\partial v_{i y}}{\partial x}+v_{i y} \frac{\partial v_{i y}}{\partial y}=-\frac{e}{m_{i}} \frac{\varphi}{\partial y}-\frac{k_{\mathrm{B}} T_{i}}{m_{i}} \frac{1}{n_{i}} \frac{\partial n_{i}}{\partial y} \\
\frac{\partial^{2} \varphi}{\partial x^{2}}+\frac{\partial^{2} \varphi}{\partial y^{2}}=4 \pi e\left(n_{e}-n_{i}\right) .
\end{gathered}
$$

Придадим плазме малое волновое возмущение с частотой $\omega$ и волновым вектором $\mathbf{k}$ :

$$
\begin{gathered}
n_{i}=n_{0 i}+\tilde{n}_{i} \exp [I(\mathbf{k r}-\omega t)], \\
\mathbf{v}_{i}=\mathbf{v}_{0 i}+\tilde{\mathbf{v}}_{i} \exp [I(\mathbf{k r}-\omega t)], \\
\varphi=\tilde{\varphi} \exp [I(\mathbf{k r}-\omega t)] .
\end{gathered}
$$

Здесь $I$ - мнимая единица. Затем перепишем возмущение в координатном представлении

$$
\begin{gathered}
n_{i}=n_{0 i}+\tilde{n}_{i} \exp \left\lfloor I\left(k_{x} x+k_{y} y-\omega t\right)\right\rfloor, \\
v_{x i}=v_{0 i}+\tilde{v}_{x i} \exp \left\lfloor I\left(k_{x} x+k_{y} y-\omega t\right)\right\rfloor, \\
\left.v_{i y}=\tilde{v}_{i y} \exp \left\lfloor I\left(k_{x} x\right)+k_{y} y-\omega t\right)\right\rfloor, \\
\left.\varphi=\tilde{\varphi} \exp \left\lfloor I\left(k_{x} x\right)+k_{y} y-\omega t\right)\right\rfloor .
\end{gathered}
$$

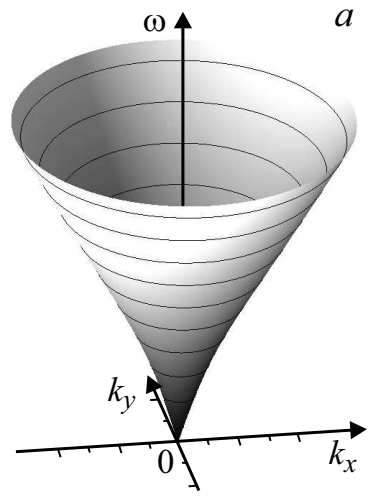

Рис. 2. Дисперсионные поверхности в декартовых координатах $\left\{k_{x}, k_{y}, \omega\right\}: a-$ при $v_{0 i}=0 ; b-$ при $v_{0 i} \neq 0$.

Подставляя (13)-(16) в систему уравнений (6)-(9), после стандартной процедуры линеаризации приходим к дисперсионному соотношению для ионно-звуковых волн

$$
\frac{\omega_{p i}^{2}}{\left(\omega-k v_{0 i}\right)^{2}}=\frac{k_{x}^{2}+k_{y}^{2}+\lambda_{D e}^{-2}}{\left(k_{x}^{2}+k_{y}^{2}\right)\left[1+\lambda_{D i}^{2}\left(k_{x}^{2}+k_{y}^{2}+\lambda_{D e}^{2}\right)\right]},
$$

в записи которого использованы обозначения: $\omega_{p i}^{2}=\frac{4 \pi e^{2} n_{0 i}}{m_{i}}-$ ионной плазменной частоты и $\lambda_{D i, e}^{2}=\frac{k_{\mathrm{B}} T_{i, e}}{4 \pi e^{2} n_{0 i, 0 e}}-$ ионной $\quad$ и электронной длины Дебая. Соотношение (18) удобно анализировать, переписав его в явной форме:

$$
\omega\left(k_{x, k_{y}}\right)=\omega_{p i} \sqrt{\frac{k_{x}^{2}+k_{y}^{2}}{k_{x}^{2}+k_{y^{2}}+\lambda_{D e}^{-2}}+\lambda_{D i}^{2}\left(k_{x}^{2}+k_{y}^{2}\right)}+v_{0 i} k_{x} .
$$

Соотношение (18) задает в декартовых координатах дисперсионную поверхность (рис. 2). При $v_{0 i}=0$ она имеет вид поверхности вращения вокруг оси $0 \omega$ (рис. 2,a).

При дисперсионная поверхность не имеет осевой симметрии и наклонена в сторону, противоположную направлению движения ионов. Например, если $v_{0 i}<0$, поверхность наклонена в положительном направлении оси $0 k_{x}$ (рис. $\left.2, b\right)$. При достаточном угле наклона на поверхности возникает локальная впадина, в окрестности которой ионно-звуковая волна может принимать форму обратной или боковой волны.

Найдем области на плоскости $\left\{k_{x}, k_{y}\right\}$, в которых угол сноса $\psi$ волны ионного звука является тупым.

Известны общие выражения для фазовой и групповой скоростей волны $[9,10]$ :

$$
\mathbf{v}_{p h}=\frac{\omega}{\mathbf{k}} \quad \text { и } \quad \mathbf{v}_{g r}=\frac{d \omega}{d \mathbf{k}}=\operatorname{grad}_{\mathbf{k}} \omega .
$$

Угол сноса $\psi$ между векторами $\mathbf{v}_{p h}$ и $\mathbf{v}_{g r}$ можно найти из векторного произведения следующим образом:

$$
\cos \psi=\frac{\left(\mathbf{v}_{p h} \mathbf{v}_{g r}\right)}{v_{p h} v_{g r}}
$$


Приведем выражения для $\mathbf{v}_{p h}$ и $\mathbf{v}_{g r}$, полученные в результате простых алгебраических вычислений:

$$
\begin{gathered}
\mathbf{v}_{p h}=\frac{1}{k_{x}^{2}+k_{y}^{2}} \\
\times\left[\omega_{p i} \sqrt{\frac{k_{x}^{2}+k_{y}^{2}}{k_{x}^{2}+k_{y}^{2} \lambda_{D e}^{-2}}+\lambda_{D i}^{2}\left(k_{x}^{2}+k_{y}^{2}\right)}+v_{0 i} k_{x}\right]\left(k_{x} \mathbf{i}+k_{y} \mathbf{j}\right), \\
\mathbf{v}_{g r}=\left\{\frac{\omega_{p i}\left[\frac{k_{x} \lambda_{D e}^{-2}}{\left(k_{x}^{2}+k_{y}^{2}+\lambda_{D e}^{-2}\right)^{2}}+k_{x} \lambda_{D i}^{2}\right]}{\left.\sqrt{\frac{k_{x}^{2}+k_{y}^{2}}{k_{x}^{2}+k_{y}^{2}+\lambda_{D e}^{-2}}+\lambda_{D i}^{2}\left(k_{x}^{2}+k_{y}^{2}\right)}+v_{0 i}\right\} \mathbf{i}}\right. \\
+\frac{\omega_{p i}\left[\frac{k_{x} \lambda_{D e}^{-2}}{\left(k_{x}^{2}+k_{y}^{2}+\lambda_{D e}^{-2}\right)^{2}}+k_{x} \lambda_{D i}^{2}\right]}{\sqrt{\frac{k_{x}^{2}+k_{y}^{2}}{k_{x}^{2}+k_{y}^{2}+\lambda_{D e}^{-2}}+\lambda_{D i}^{2}\left(k_{x}^{2}+k_{y}^{2}\right)} \mathbf{j},}
\end{gathered}
$$

где $\mathbf{i}$ и $\mathbf{j}-$ единичные векторы в плоскости $\left\{k_{x}, k_{y}\right\}$.

Полезно привести значение линейной скорости ионного звука, определяемой как

$$
c_{s}=\lim _{k \rightarrow 0} \frac{\omega}{k}=\lim _{k \rightarrow 0} \frac{d \omega}{d k} .
$$

Оно также будет зависеть от направления. Приведем значение $c_{S}$ в зависимости от угла $\theta$ между вектором $\mathbf{k}$ и направлением дрейфа ионов

$$
c_{S}=\omega_{p i} \sqrt{\lambda_{D i}^{2}+\lambda_{D e}^{2}}+v_{0 i} \cos \theta .
$$

На рис. 3 представлен график $c_{S}(\omega)$ в полярных координатах.

Были проведены вычисления угла сноса по формулам (20)-(22). Результаты вычислений удобно показать

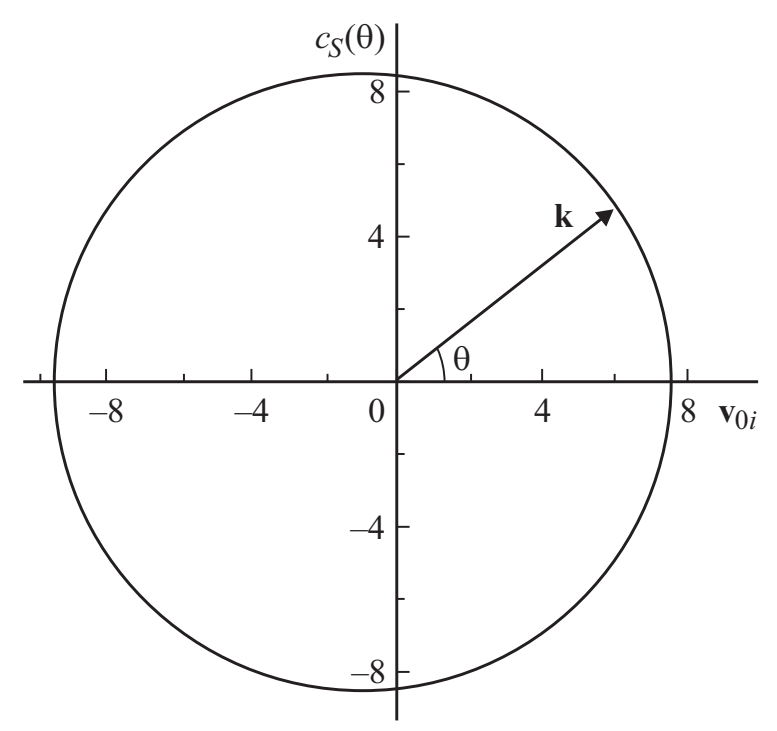

Рис. 3. График в полярных координатах.
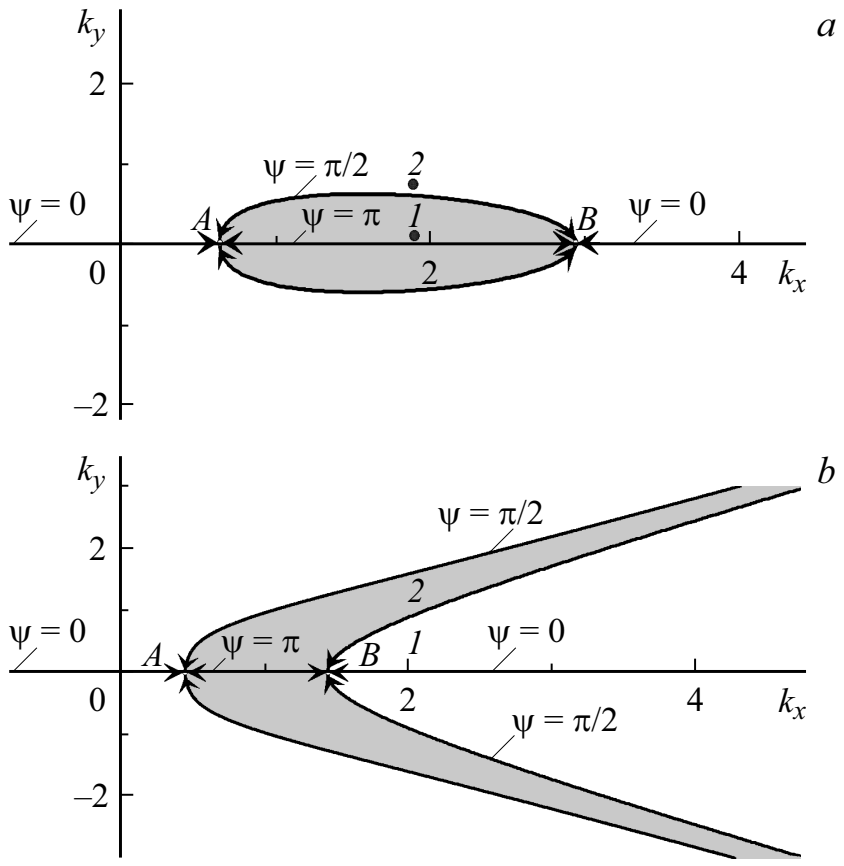

Рис. 4. Области в плоскости $\left\{k_{x}, k_{y}\right\}$, в которой угол $\psi$ является тупым: $a-$ при $v_{0 i}-0.95 ; b-$ при $v_{0 i}=-1.2$.

графически. На рис. 4 показаны области в плоскости $\left\{k_{x}, k_{y}\right\}$, в которой угол $\psi$ является тупым. При различных значениях скорости $v_{0 i}$ форма области тупых углов $\psi$ может иметь различный вид. Так, при

$$
v_{0 i}<V_{1}=4 \omega_{p i} \lambda_{D i} \sqrt{\frac{\delta(\delta \sqrt{4 \delta+3}+2 \delta+1)}{(\sqrt{4 \delta+3}+2 \delta)^{3}}},
$$

где $\delta=\frac{\lambda_{D i}}{\lambda_{D e}}$, такой области нет вообще, при

$$
V_{1}<v_{0 i}<V_{2}=\omega_{p i} \lambda_{D i}
$$

граница существует, она замкнута и ограничивает конечную яйцеобразную область (рис. 4, $a$ ), приходящуюся на ионно-плазменные колебания, однако при $v_{0 i}>V_{2}$ граница разомкнута, она ограничивает бесконечную область в форме бумеранга (рис. 4, $b$ ). Значения критических скоростей $V_{1}$ и $V_{2}$ ранее получены в [3]. Границы областей отделяются кривыми, для которых значение угла сноса $\psi= \pm \pi / 2$. Выколотые точки $A$ и $B$ дают неопределенность в значениях угла сноса, так значения групповой скорости в них обращаются в нуль.

\section{Нелинейная теория}

В нелинейной теории будем исходить из тех же уравнений ионной динамики в плазме, что и предыдущем разделе. Далее перейдем к новой переменной: $\xi=l_{x} x+l_{y} y-V t$, где $l_{x}^{2}+l_{y}^{2}=1, l_{x, y}$ - направляющие косинусы вектора $\mathbf{k}$. Тогда уравнения в частных произ- 

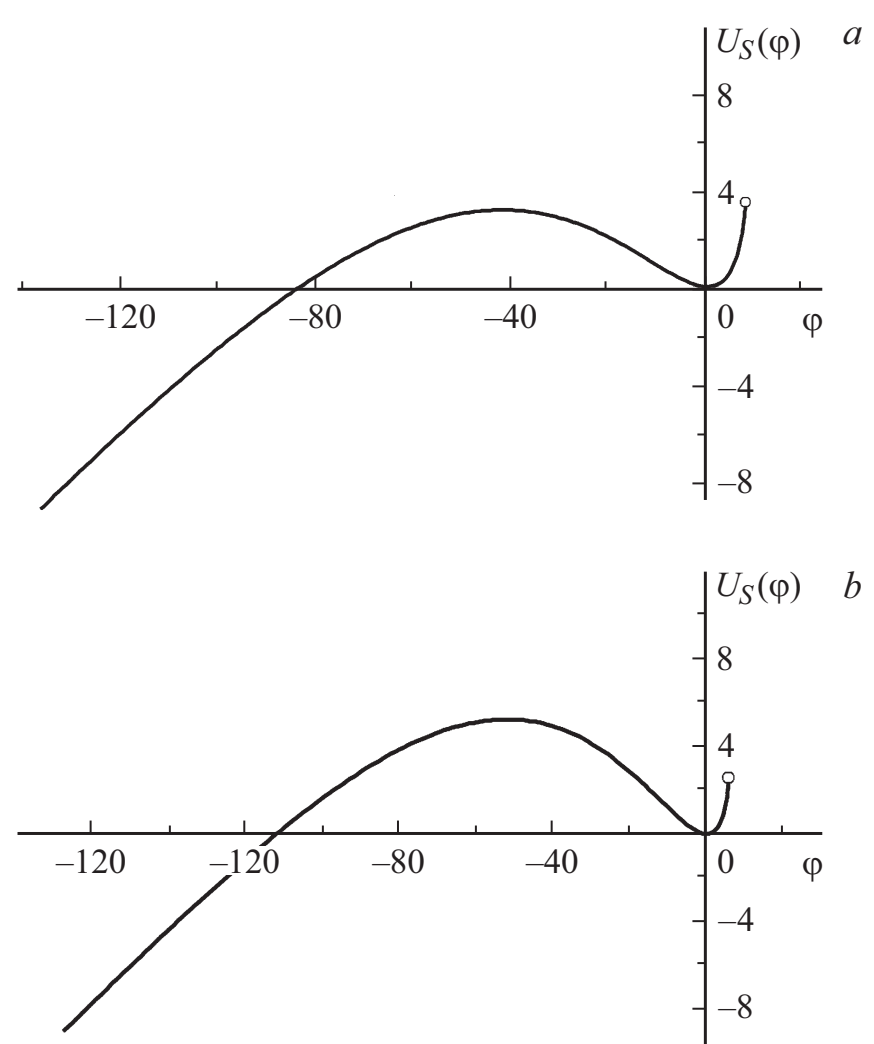

Рис. 5. Графики псевдопотенциала (44) для ионно-звуковой волны, распространяющихся под углами: $a-\theta=1.75^{\circ} ; b-$ $\theta=24^{\circ}$.
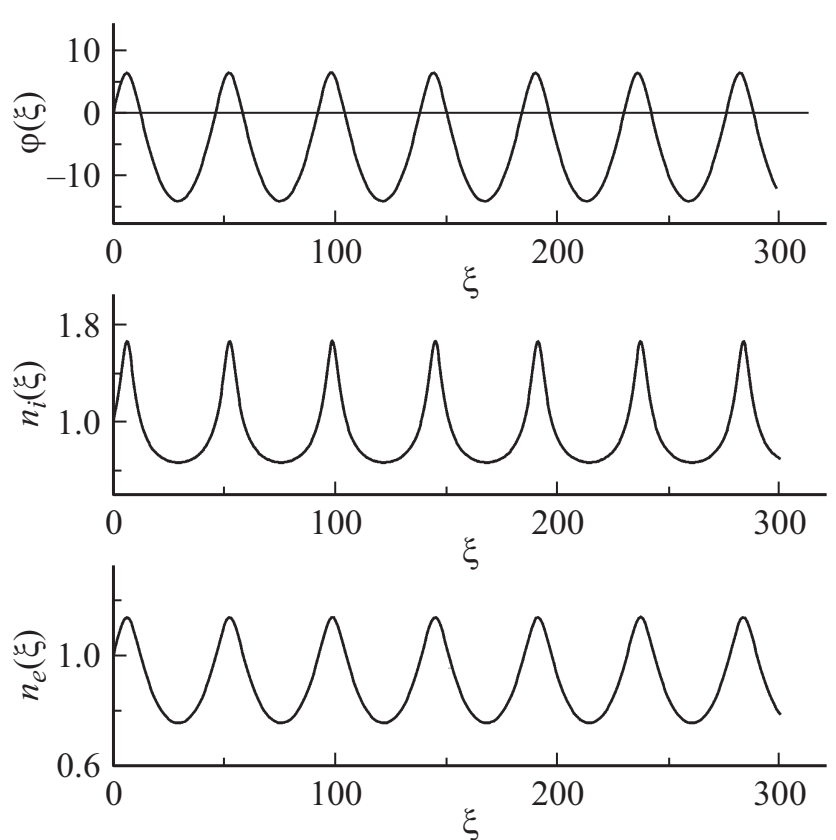

Рис. 6. Профили ионно-звуковой волны, распространяющейся под углом $\theta=1.75^{\circ}$. водных (6)-(9) перепишутся в виде системы обыкновенных дифференциальных уравнений

$$
\begin{gathered}
\frac{d}{d \xi}\left[n_{i}\left(-V+l_{x} v_{i x}+l_{y} v_{i y}\right)\right]=0, \\
-V \frac{d v_{i x}}{d \xi}+l_{x} v_{i x} \frac{d v_{i x}}{d \xi}+l_{y} v_{i y} \frac{d v_{i x}}{d \xi} \\
=-\frac{e}{m_{i}} l_{x} \frac{d \varphi}{d \xi}-\frac{k_{\mathrm{B}} T_{i}}{m_{i}} l_{x} \frac{1}{n_{i}} \frac{d n_{i}}{d \xi}, \\
-V \frac{d v_{i y}}{d \xi}+l_{x} v_{i x} \frac{d v_{i y}}{d \xi}+l_{y} v_{i y} \frac{d v_{i y}}{d \xi} \\
=-\frac{e}{m_{i}} l_{y} \frac{d \varphi}{d \xi}-\frac{k_{\mathrm{B}} T_{i}}{m_{i}} l_{y} \frac{1}{n_{i}} \frac{d n_{i}}{d \xi} \\
\frac{d^{2} \varphi}{d \xi^{2}}=4 \pi e\left[n_{e}(\varphi)-n_{i}\right] .
\end{gathered}
$$

Эти уравнения совместно с начальными условиями $n_{i}(0)=n_{0 i}, v_{i x}(0)=v_{0 i}, v_{i y}(0), \varphi(0)=0$ образуют нелинейную задачу Коши.

Проведем нормировку задачи следующим образом: $\xi$ нормируем на $\lambda_{D i} \sqrt{\frac{k_{\mathrm{B}} T_{i}}{4 \pi e^{2} n_{01}}}$, все скорости - на $\omega_{i} \lambda_{D i}$, все концентрации - на $n_{0 i}$, а электростатический потенциал $\varphi-$ на $\frac{k_{\mathrm{B}} T_{i}}{e}$. Тогда задача Коши примет вид

$$
\begin{gathered}
\frac{d}{d \xi}\left[n_{i}\left(-V+l_{x} v_{i x}+l_{y} v_{i y}\right)\right]=0 \\
-V \frac{d v_{i x}}{d \xi}+l_{x} v_{i x} \frac{d v_{i x}}{d \xi}+l_{y} v_{y} \frac{d v_{i x}}{d \xi}=-l_{x} \frac{d \varphi}{d \xi}-l_{x} \frac{1}{n_{i}} \frac{d n_{i}}{d \xi} \\
-V \frac{d v_{i y}}{d \xi}+l_{x} v_{i x} \frac{d v_{i y}}{d \xi}+l_{y} v_{y} \frac{d v_{i y}}{d \xi}=-l_{y} \frac{d \varphi}{d \xi}-l_{y} \frac{1}{n_{i}} \frac{d n_{i}}{d \xi}, \\
\frac{d^{2} \varphi}{d \xi^{2}}=n_{e n}(\varphi)-n_{i}, \\
n_{i}(0)=1 ; \quad v_{i x}(0)=v_{0 i} ; \quad v_{i y}(0)=0 ; \quad \varphi(0)=0,
\end{gathered}
$$
где $n_{e}(\varphi)=\exp (\tau \varphi), \tau=T_{i} / T_{e}$.

Проинтегрируем уравнение непрерывности (31) с учетом (35) и выразим из получившегося уравнения $v_{i y}$ :

$$
v_{i y}=\frac{1}{l_{y}}\left(V-l_{x} v_{i x}+\frac{l_{x} v_{0 i}-V}{n_{i}}\right) .
$$

Далее подставим (36) в уравнения (32) и (33):

$$
\begin{aligned}
& -v_{i y} \frac{d v_{i x}}{d \xi}+l_{x} v_{i x} \frac{d v_{i x}}{d \xi}+\left(V-l_{x} v_{i x}+\frac{l_{x} v_{0 i}-V}{n_{i}}\right) \frac{d v_{i x}}{d \xi} \\
& =-l_{x} \frac{d \varphi}{d \xi}-l_{x} \frac{1}{n_{i}} \frac{d n_{i}}{d \xi}, \\
& \frac{1}{l_{y}} \frac{l_{x} v_{0 i}-V}{n_{i}} \frac{d\left(\frac{l_{x} v_{0 i}-V}{n_{i}}-l_{x} v_{i x}\right)}{d \xi}=-l_{y} \frac{d \varphi}{d \xi}-l_{y} \frac{1}{n_{i}} \frac{d n_{i}}{d \xi} .
\end{aligned}
$$




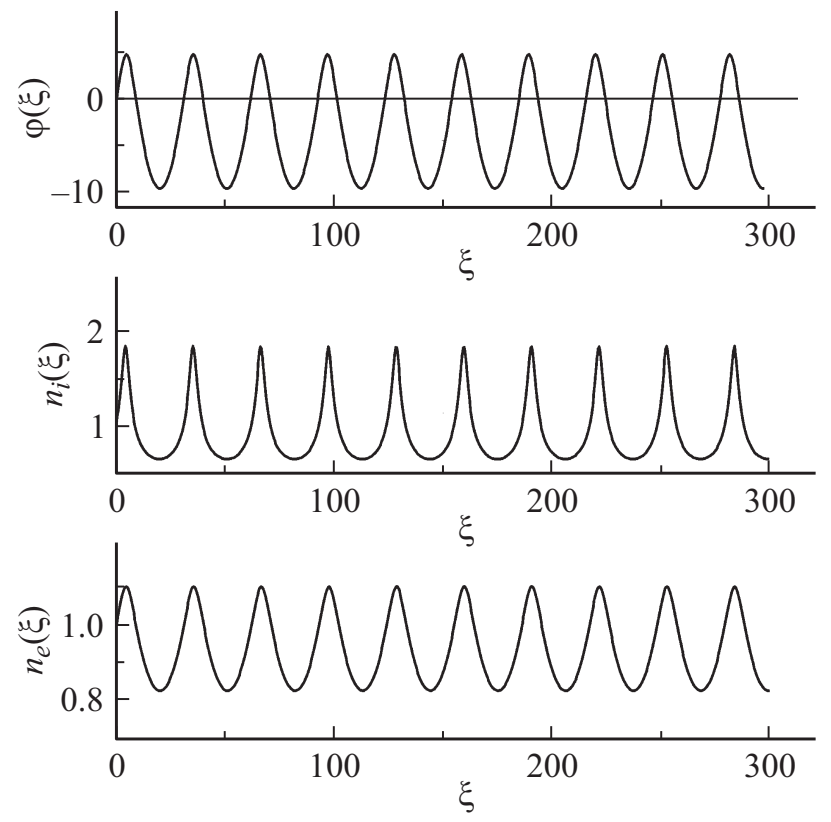

Рис. 7. Профили ионно-звуковой волны, распространяющейся под углом $\theta=24^{\circ}$.

Выразим из (37) $\frac{d v_{i x}}{d \xi}$

$$
\frac{d v_{i x}}{d \xi}=-\frac{l_{x} n_{i}}{l_{x} v_{0 i}-V}\left(\frac{d \varphi}{d \xi}+\frac{1}{n_{i} \frac{d n_{i}}{d \xi}}\right)
$$

и подставим затем (39) в уравнение (38)

$$
\begin{aligned}
& \frac{1}{l_{y}} \frac{l_{x} v_{0 i}-V}{n_{i}} \frac{d}{d \xi}\left(\frac{l_{x} v_{0 i}-V}{n_{i}}\right)+\frac{l_{x}^{2}}{l_{y}}\left(\frac{d \varphi}{d \xi}+\frac{1}{n_{i}} \frac{d n_{i}}{d \xi}\right) \\
& =-l_{y} \frac{d \varphi}{d \xi}-l_{y} \frac{1}{n_{i}} \frac{d n_{i}}{d \xi} .
\end{aligned}
$$

В результате интегрирования (40) получим

$$
\xi=\ln \left(\frac{1}{n_{i}}\right)+\frac{1}{2}\left[\left(\frac{l_{x} v_{0 i}-V}{2}\right)^{2}-\left(\frac{l_{x} v_{0 i}-V}{n_{i}}\right)^{2}\right] .
$$

Далее необходимо выразить $n_{i}(\varphi)$ из уравнения (41), решив при этом трансцендентное уравнение. Сделать это можно с помощью специальной функции - $W$ функции Ламберта [11]:

$n_{i}(\varphi)=\sqrt{-\frac{\left(l_{x} v_{0 i}-V\right)^{2}}{W_{0,-1}\left\{-\left(l_{x} v_{0 i}-V\right)^{2} \exp \left[-\frac{\left(l_{x} v_{0 i}-V\right)}{2}+2 \varphi\right]\right\}}}$.

Подставим (1) и (42) в уравнение Пуассона, домножим его на $\frac{d \varphi}{d \xi}$ и проинтегрируем. Тогда получим

$$
\frac{1}{2}\left(\frac{d \varphi}{d \xi}\right)^{2}=E-U_{S}(\varphi)
$$

где $E-$ постоянная интегрирования, а $U_{S}(\varphi)-$ псевдопотенциал Сагдеева, имеющий следующий вид:

$$
\begin{aligned}
& U_{S}(\varphi)=\left|l_{x} v_{0 i}-V\right| \\
& \times \frac{W_{0,-1}\left\{-\left(l_{x} v_{0 i}-V\right)^{2} \exp \left[-\frac{\left(l_{x} v_{0 i}-V\right)^{2}}{2}+2 \varphi\right]\right\}-1}{\sqrt{-W_{0,-1}\left\{-\left(l_{x} v_{0 i}-V\right)^{2} \exp \left[-\frac{\left(l_{x} v_{0 i}-V\right)^{2}}{2}+2 \varphi\right]\right\}}} \\
& -\frac{\exp (\tau \varphi)}{\tau}+C
\end{aligned}
$$

в котором постоянную интегрирования удобно выбирать из условия $U_{S}(0)=0$, а номер действительной ветви $W$ функции Ламберта - из условия квазинейтральности невозмущенной плазмы [12]. В нашем случае для периодической волны следует выбирать - 1-ую ветвь.

Графики псевдопотенциала (44) для двух случаев ионно-звуковой волны, распространяющихся под углами $24^{\circ}$ и $\theta=1.75^{\circ}$ для следующих параметров $\tau=0.2$, $v_{0 i}=-0.95, V=3.5$ и $C=66.2$, представлены на рис. 5. Для угла $\theta=175^{\circ}$ угол сноса $\psi \approx 176.5^{\circ}$, т.е. ионно-звуковая волна является почти обратной, а для угла $\theta=24^{\circ}$ в волне $\mathbf{v}_{p h}$ и $\mathbf{v}_{g r}$ почти перпендикулярны друг другу. Эти два случая помечены кружочками 1 и 2 на рис. $4, a$.

Оба псевдопотенциала на рис. 5 имеют глобальные минимумы в начале координат. Профили ионно-звуковой волны для этих случаев были вычислены и представлены на рис. 6 и 7. Эти профили ничем не отличаются от профилей обычных прямых ионно-звуковых волн, показанных, например, в [13].

\section{Заключение}

Рассмотрены наклонные ионно-звуковые волны и плазме, в которой ионы движутся однонаправлено. В рамках линейной теории выведено двумерное дисперсионное соотношение и показано, что в системе возможны обратные и боковые волны.

Развитая нелинейная теория обратных и боковых волн базируется на выводе и анализе псевдопотенциала Сагдеева. Вычислялись нелинейные профили обратной и боковой ионно-звуковой волны. Найдено, что профили обратной и боковой волн неотличимы от профиля обычной прямой ионно-звуковой волны. Указанные волны можно различить только по кинематическим характеристикам.

\section{Конфликт интересов}

Авторы заявляют, что у них нет конфликта интересов.

\section{Список литературы}

[1] Dubinov A.E., Kitayev I.N. // Phys. Wave Phenomena. 2017. Vol. 25. P. 137.

[2] Dubinov A.E., Kitayev I.N. // Phys. Plasmas. 2016. Vol. 23. P. 104503. 
[3] Dubinov A.E., Kitayev I.N. // Contrib. Plasma Phys. 2017. Vol. 57. P. 373.

[4] Gil'man O.L., Ostrovsky L.A., Feinstein S.M. // IEEE Trans. Plasma Sci. 1981. Vol. 9. P. 286.

[5] Yashvir Tiwari R.S., Sharma S.R. // Can. J. Phys. 1988. Vol. 66. P. 824.

[6] Nakamura Y., Ohtani K. // J. Plasma Phys. 1995. Vol. 53. P. 235.

[7] El-Labany S.K. // J. Plasma Phys. 1995. Vol. 54. P. 285.

[8] Dubinin E., Sauer K., McKenzie J.F. // J. Geophys. Res. 2004. Vol. 109. P. A02208.

[9] Ginzburg V.L. The Propagation of Electromagnetic Waves in Plasmas. Pergamon, London: Addison-Wesley, Reading, Mass, 1964.

[10] Agranovich V.M., Ginzburg V.L. Crystal Optics with Spatial Dispersion and Excitons. Berlin: Springer-Verlag, 1984.

[11] Dubinov A.E., Dubinova I.D. // J. Plasma Phys. 2005. Vol. 71. P. 715 .

[12] Gordienko V.A., Dubinova I.D., Dubinov A.E. // Plasma Phys. Rep. 2006. Vol. 32. P. 910.

[13] Dubinov A.E. // J. Appl. Mech. Techn. Phys. 2007. Vol. 48. P. 621. 